\title{
Electromagnetic response of hot QCD medium in the presence of background time-varying fields
}

\author{
Gowthama K K, ${ }^{*}$ Manu Kurian ${ }^{\dagger}{ }^{\dagger}$ and Vinod Chandra ${ }^{\ddagger}$ \\ Indian Institute of Technology Gandhinagar, Gandhinagar-382355, Gujarat, India
}

(Received 20 August 2021; accepted 1 November 2021; published 29 November 2021)

\begin{abstract}
The response of the hot QCD medium in the presence of external time dependent electromagnetic fields has been studied within the relativistic Boltzmann transport theory. The impact of the time dependence of the electromagnetic fields and collisional aspects of the medium to the induced electric and Hall current densities has been explored. The nonequilibrium momentum distribution of degrees of freedom has been obtained in the presence of space-time varying electromagnetic fields. Further, the analysis has been extended to an anisotropic QCD medium while incorporating the in-medium interaction effects. It is observed that the electric charge transport is sensitive to the inhomogeneity of the fields and the momentum anisotropy of the QCD medium.
\end{abstract}

DOI: 10.1103/PhysRevD.104.094037

\section{INTRODUCTION}

Experiments at Relativistic Heavy Ion Collider (RHIC) and Large Hadron Collider (LHC) have provided evidence for the existence of strongly interacting matter-quark gluon plasma (QGP) [1]. Transport coefficients of the hot QCD matter serve as the input parameters for the hydrodynamical description of the evolution of the created medium and act as a key ingredient to explore critical properties of the medium [2]. Recent observations at the RHIC and LHC have indicated the presence of a strong magnetic field in the early stages of heavy-ion collision $[3,4]$. Even though there is no definitive description of the evolution of the generated magnetic field, various models predict that the magnetic field may persist for much longer in the QGP due to the backreaction in the medium [5-9]. This persisting magnetic field may influence the behavior of the hot QCD matter and hence is pertinent to study the various properties of the QCD medium in the presence of magnetic fields.

The electric charge transport in the hot deconfined quark matter has gained much attention due to the generated electromagnetic fields in the collision experiments. The medium response to the external electromagnetic fields can be studied in terms of the induced electric and Hall current densities and associated conductivities. The generated electric field in asymmetric heavy-ion collisions has a

\footnotetext{
"gowthama@iitgn.ac.in manu.kurian@iitgn.ac.in

*vchandra@iitgn.ac.in
}

Published by the American Physical Society under the terms of the Creative Commons Attribution 4.0 International license. Further distribution of this work must maintain attribution to the author(s) and the published article's title, journal citation, and DOI. Funded by SCOAP ${ }^{3}$. preferred direction, and the conductivities may strongly depend on the charge asymmetric flow [10]. The study of electromagnetic responses on the QCD medium is also relevant in the context of chiral magnetic effect [11] and emission rate of soft photons [12] in the heavy-ion collisions. Electric charge transport has been studied in the presence of constant electric and magnetic fields in the regimes where the strengths of the fields are weak [13-18] and in the strong field limit [19-22]. Attributing to the fact that the generated electromagnetic fields are varying with space and time [5,23-26], it is important to investigate the QCD medium response to the inhomogeneous fields. This sets the motivation of the current study.

Electrical conductivity of the hot QCD medium has been investigated in several studies within transport theory [27-29], Kubo formalism [30] and lattice QCD estimations $[31,32]$. Recently, we have estimated the additional component to the current density due to the time dependence of the external electric field [33]. The focus of the current investigation is to extend the analysis to develop a general framework to study the response of the QCD medium to a time-varying electric and magnetic field. To that end, we have obtained the general form of the near-equilibrium quark degrees of freedom in the presence of inhomogeneous fields within the transport theory. We have also explored the electric charge transport of an anisotropic weakly magnetized QCD medium. The in-medium interactions have been incorporated in the analysis by adopting a recently developed effective fugacity quasiparticle model (EQPM) [34]. We have analyzed the impact of time dependence of the fields and momentum anisotropy of the medium to the electric charge transport process along with the effects of mean-field corrections to the respective current densities. 
This may perhaps be the first attempt where the physics of inhomogeneity of the external electromagnetic fields has been incorporated in the analysis of electric charge transport in the context of QCD medium. Our estimations are consistent with the studies so far, as we have correctly reproduced the earlier results within the general formalism with the choice of appropriate electromagnetic fields.

Notations and conventions: The subscript $k$ denotes the particle species. The quantity $q_{f_{k}}$ is the electric charge of the particle with flavor $f$ of the $k$ th species. The particle velocity is defined as $\mathbf{v}=\frac{\mathbf{p}}{\epsilon}$, where $\mathbf{p}$ is the momentum and $\epsilon=\sqrt{p^{2}+m_{f}^{2}}$ is the energy (with $m_{f}$ as the mass of quark with flavor $f$ ) of the particle. The component of a three vector $\mathbf{A}$ is denoted with the Latin indices $A^{i}$. The quantities $E=|\mathbf{E}|$ and $B=|\mathbf{B}|$ denote the magnitude of the electric and magnetic fields.

\section{ELECTRIC CHARGE TRANSPORT IN QCD MEDIUM WITH TIME-VARYING FIELDS}

The response of the QCD medium to the external electromagnetic fields can be studied with induced current densities and the associated conductivities. The general form of the induced vector current in the QCD medium with a nonvanishing quark chemical potential $\mu$ in terms of quark and antiquark momentum distribution function $f_{k}=f_{k}^{0}+\delta f_{k}$ is as follows,

$$
\mathbf{j}=2 N_{c} \sum_{f} \int d P \mathbf{v}\left(q_{q} f_{q}-q_{\bar{q}} f_{\bar{q}}\right)
$$

where $v_{i}$ is the component of velocity and $d P=\frac{d^{3} \mathbf{p}}{(2 \pi)^{3}}$. The flavor summation (over the up, down, and strange quarks) arises from the degeneracy factor $2 N_{c} \sum_{f}$ of the quarks/antiquarks with $N_{c}$ number of colors. To study the impact of electromagnetic fields on the current density, one needs to explore the nonequilibrium part of the distribution function. We proceed further to find the near-equilibrium distribution function by solving the transport equation within the relaxation time approximation. The Boltzmann equation that describes the dynamics of the distribution function in the presence of an external electromagnetic field has the following form,

$$
\frac{\partial f_{k}}{\partial t}+\mathbf{v} \cdot \frac{\partial f_{k}}{\partial \mathbf{x}}+q_{f_{k}}[\mathbf{E}+\mathbf{v} \times \mathbf{B}] \cdot \frac{\partial f_{k}}{\partial \mathbf{p}}=-\frac{\delta f_{k}}{\tau_{R}},
$$

where $\tau_{R}$ is the relaxation time [35]. We solve the relativistic Boltzmann equation by assuming the following ansatz for $\delta f_{k}$ due to the inhomogeneous electromagnetic fields,

$$
\delta f_{k}=(\mathbf{p} . \boldsymbol{\Xi}) \frac{\partial f_{k}^{0}}{\partial \epsilon},
$$

where the vector $\boldsymbol{\Xi}$ is related to the strength of the electromagnetic field and its first-order (leading order) spacetime derivatives, with the following form,

$$
\begin{aligned}
\boldsymbol{\Xi}= & \alpha_{1} \mathbf{E}+\alpha_{2} \dot{\mathbf{E}}+\alpha_{3}(\mathbf{E} \times \mathbf{B})+\alpha_{4}(\dot{\mathbf{E}} \times \mathbf{B})+\alpha_{5}(\mathbf{E} \times \dot{\mathbf{B}}) \\
& +\alpha_{6}(\boldsymbol{\nabla} \times \mathbf{E})+\alpha_{7} \mathbf{B}+\alpha_{8} \dot{\mathbf{B}}+\alpha_{9}(\boldsymbol{\nabla} \times \mathbf{B}) .
\end{aligned}
$$

Here, $\alpha_{i}(i=(1,2, \ldots, 9))$ are the unknown functions that relate to the respective electric charge transport coefficients and can be obtained by the microscopic description of the QCD medium. The present focus is on the case with chiral chemical potential is zero and hence $\alpha_{i}=0$ for $i=(6,7,8)$ considering the parity symmetry in the analysis [36]. Employing Eq. (3) and Eq. (4) in Eq. (2) we have,

$$
\begin{aligned}
& \epsilon \mathbf{v} \cdot\left[\alpha_{1} \dot{\mathbf{E}}+\dot{\alpha}_{1} \mathbf{E}+\alpha_{2} \ddot{\mathbf{E}}+\dot{\alpha}_{2} \dot{\mathbf{E}}+\alpha_{3}(\dot{\mathbf{E}} \times \mathbf{B})+\alpha_{3}(\mathbf{E} \times \dot{\mathbf{B}})+\dot{\alpha}_{3}(\mathbf{E} \times \mathbf{B})+\alpha_{4}(\dot{\mathbf{E}} \times \dot{\mathbf{B}})+\alpha_{4}(\ddot{\mathbf{E}} \times \mathbf{B})+\dot{\alpha}_{4}(\dot{\mathbf{E}} \times \mathbf{B})\right. \\
& \left.+\alpha_{5}(\dot{\mathbf{E}} \times \dot{\mathbf{B}})+\alpha_{5}(\mathbf{E} \times \ddot{\mathbf{B}})+\dot{\alpha}_{5}(\mathbf{E} \times \dot{\mathbf{B}})+\alpha_{9}(\boldsymbol{\nabla} \times \dot{\mathbf{B}})+\dot{\alpha}_{9}(\boldsymbol{\nabla} \times \mathbf{B})\right]+q_{f_{k}} \mathbf{v} \cdot \mathbf{E}-\alpha_{1} q_{f_{k}} \mathbf{v} \cdot(\mathbf{E} \times \mathbf{B})-\alpha_{2} q_{f_{k}} \mathbf{v} \cdot(\dot{\mathbf{E}} \times \mathbf{B}) \\
& +\alpha_{3} q_{f_{k}}(\mathbf{v} \cdot \mathbf{E})\left(B^{2}\right)-\alpha_{3} q_{f_{k}}(\mathbf{v} . \mathbf{B})(\mathbf{B} . \mathbf{E})+\alpha_{4} q_{f_{k}}(\mathbf{v} \cdot \dot{\mathbf{E}})\left(B^{2}\right)-\alpha_{4} q_{f_{k}}(\mathbf{v} \cdot \mathbf{B})(\mathbf{B} \cdot \dot{\mathbf{E}})+\alpha_{5} q_{f_{k}}(\mathbf{v} \cdot \mathbf{E})(\dot{\mathbf{B}} \cdot \mathbf{B})-\alpha_{5} q_{f_{k}}(\dot{\mathbf{B}} \cdot \mathbf{v})(\mathbf{E} . \mathbf{B}) \\
& -\alpha_{9} q_{f_{k}}(\mathbf{B} \cdot v)(\boldsymbol{\nabla} \cdot \mathbf{B})=-\frac{\epsilon}{\tau_{R}}\left[\alpha_{1} \mathbf{v} \cdot \mathbf{E}+\alpha_{2} \mathbf{v} \cdot \dot{\mathbf{E}}+\alpha_{3} \mathbf{v} \cdot(\mathbf{E} \times \mathbf{B})+\alpha_{4} \mathbf{v} \cdot(\dot{\mathbf{E}} \times \mathbf{B})+\alpha_{5} \mathbf{v} \cdot(\mathbf{E} \times \dot{\mathbf{B}})+\alpha_{9} \mathbf{v} \cdot(\boldsymbol{\nabla} \times \mathbf{B})\right] .
\end{aligned}
$$

We consider the terms with first-order derivatives of the fields and neglect higher-order derivative terms as the electromagnetic fields vary slowly in space and time to incorporate the collisional aspects of the QCD medium. Hence, the terms with $\dot{\alpha}_{2}, \dot{\alpha}_{4}, \dot{\alpha}_{5}, \dot{\alpha_{9}}$ are neglected in the current analysis. Incorporating this approximation and comparing the coefficients of terms with the same tensorial structure in both sides of Eq. (5), we obtain the coupled differential equations as follows,

$$
\begin{aligned}
& \dot{\alpha_{1}}=-\left[\frac{1}{\tau_{R}} \alpha_{1}+\left(\frac{q_{f k} B^{2}}{\epsilon}-\frac{\tau_{R} q_{f k} B \dot{B}}{\epsilon}\right) \alpha_{3}+\frac{q_{f k}}{\epsilon}\right], \\
& \dot{\alpha_{3}}=-\frac{1}{\tau_{R}} \alpha_{3}+\frac{q_{f k}}{\epsilon} \alpha_{1},
\end{aligned}
$$

along with the coupled equations, 


$$
\begin{aligned}
& \alpha_{2}=-\tau_{R}\left[\alpha_{1}+\frac{q_{f_{k}} \alpha_{4} B^{2}}{\epsilon}\right], \quad \alpha_{5}=-\tau_{R} \alpha_{3}, \\
& \alpha_{4}=-\tau_{R}\left[\alpha_{3}-\frac{\alpha_{2} q_{f_{k}}}{\epsilon}\right] .
\end{aligned}
$$

with $\dot{B}=|\dot{\mathbf{B}}|$. The coupled differential equations can be described in terms of matrix equation as follows,

$$
\frac{d X}{d t}=A X+G
$$

where the matrices can be defined as follows,

$$
X=\left(\begin{array}{l}
\alpha_{1} \\
\alpha_{3}
\end{array}\right), \quad A=\left(\begin{array}{cc}
-\frac{1}{\tau_{R}} & -\frac{q_{f_{k}} F^{2}}{\epsilon} \\
\frac{q_{f_{k}}}{\epsilon} & -\frac{1}{\tau_{R}},
\end{array}\right), \quad G=\left(\begin{array}{c}
\frac{-q_{f_{k}}}{\epsilon} \\
0
\end{array}\right),
$$

with $F=\sqrt{B\left(B-\tau_{R} \dot{B}\right)}$. We solve Eq. (8) by finding the solution of the homogeneous equation $\frac{d X}{d t}=A X$ by diagonalizing the matrix $A$. Then, we find the solution to the nonhomogeneous part of Eq. (8) by replacing the constants of integration with time dependent functions $k_{1}(t)$ and $k_{2}(t)$. Using the method of variation of constants, we obtain the particular solution as follows,

$$
\alpha_{1}=k_{1} i F e^{\eta_{1}}-k_{2} i F e^{\eta_{2}}, \quad \alpha_{3}=k_{1} e^{\eta_{1}}+k_{2} e^{\eta_{2}}
$$

The functions $k_{1}(t)$ and $k_{2}(t)$ can be defined as $k_{1}=\frac{i q_{f_{k}}}{2 \epsilon} I_{1}$ and $k_{2}=-\frac{i q_{f_{k}}}{2 \epsilon} I_{2}$ where $\eta_{j}$ and $I_{j},(j=1,2)$ take the following forms,

$$
\eta_{j}=-\frac{t}{\tau_{R}}+a_{j} \frac{q_{f_{k}} i}{\epsilon} \int F d t, \quad I_{j}=\int \frac{e^{-\eta_{j}}}{F},
$$

with $a_{1}=1$ and $a_{2}=-1$. Substituting Eq. (10) in Eq. (9) and employing Eq. (7), we obtain the master equation for $\alpha_{i}$ as follows,

$$
\begin{gathered}
\alpha_{1}=-\frac{\tilde{\Omega}_{k}}{2}\left(I_{1} e^{\eta_{1}}+I_{2} e^{\eta_{2}}\right), \\
\alpha_{3}=\frac{q_{f k} i}{2 \epsilon}\left(I_{1} e^{\eta_{1}}-I_{2} e^{\eta_{2}}\right), \\
\alpha_{5}=-\frac{\tau_{R} q_{f k} i}{2 \epsilon}\left(I_{1} e^{\eta_{1}}-I_{2} e^{\eta_{2}}\right), \\
\alpha_{2}=\frac{\left(\frac{\tilde{\Omega}_{k} \tau_{R}}{2}+\frac{i \Omega_{k}^{2} \tau_{R}^{2}}{2}\right) I_{1} e^{\eta_{1}}+\left(\frac{\tilde{\Omega}_{k} \tau_{R}}{2}-\frac{i \Omega_{k}^{2} \tau_{R}^{2}}{2}\right) I_{2} e^{\eta_{2}}}{1+\Omega_{k}^{2} \tau_{R}^{2}} \\
\alpha_{4}=\frac{-\left(\frac{\tilde{\Omega}_{k}^{2} \tau_{R}^{2}}{2 F}+\frac{i q_{f k} \tau_{R}}{2 \varepsilon}\right) I_{1} e^{\eta_{1}}-\left(\frac{\tilde{\Omega}_{k}^{2} \tau_{R}^{2}}{2 F}-\frac{i q_{f k} \tau_{R}}{2 \varepsilon}\right) I_{2} e^{\eta_{2}}}{1+\Omega_{k}^{2} \tau_{R}^{2}}
\end{gathered}
$$

with $\Omega_{k}=\frac{q_{f k} B}{\epsilon}$ represents the cyclotron frequency at finite $B$ and $\tilde{\Omega}_{k}=\frac{q_{f k} F}{\varepsilon}$ for the time-varying magnetic field. Now, we solve the master equation of $\alpha_{i}$ for various choices of the electromagnetic fields $\mathbf{E}$ and $\mathbf{B}$.

\section{A. Case I: Constant electric and magnetic fields}

For the case of constant electric and magnetic field, the terms with $\alpha_{1}$ and $\alpha_{3}$ will give nonzero contributions to the current density as the terms associated with space-time derivatives of the fields vanish as described in Eq. (3). Solving $\alpha_{1}$ and $\alpha_{3}$ from Eq. (11) and Eq. (12) for constant electromagnetic fields, we obtain

$\alpha_{1}=\frac{-\epsilon q_{f_{k}}}{\tau_{R}\left[\left(\frac{\epsilon}{\tau_{R}}\right)^{2}+\left(q_{f_{k}} B\right)^{2}\right]}, \quad \alpha_{3}=\frac{-q_{f_{k}}^{2}}{\left[\left(\frac{\epsilon}{\tau_{R}}\right)^{2}+\left(q_{f_{k}} B\right)^{2}\right]}$.

Employing Eq. (3) in Eq. (1), we obtain $j^{i}=\sigma_{e} \delta^{i j} E_{j}+$ $\sigma_{H} \epsilon^{i j} E_{j}$ with $\sigma_{e}$ and $\sigma_{H}$ denote the electrical and Hall conductivities, respectively. The results obtained are in agreement with the observations of Ref. [13]. It is important to emphasize that the Hall current vanishes at $\mu=0$ case. In the limit of vanishing magnetic field, we obtain the electric current as,

$$
\mathbf{j}=\frac{\mathbf{E}}{3} 2 N_{c} \sum_{k} \sum_{f}\left(q_{f_{k}}\right)^{2} \int d P \tau_{R} \frac{p^{2}}{\epsilon^{2}}\left(-\frac{\partial f_{k}^{0}}{\partial \epsilon}\right)
$$

which agrees with the results of [29]. However, in the presence of a strong uniform magnetic field, the longitudinal electrical conductivity has the dominant role in the electric charge transport and $\sigma_{H} \sim 0$ as the transverse transport to the magnetic field is negligible due to the $1+1-\mathrm{D}$ Landau dynamics of quarks and antiquarks.

\section{B. Case II: Response of weakly magnetized medium to time dependent electric field}

We consider the electric charge transport process in the presence of a time-varying electric field in a weakly magnetized QCD medium. Hence, the term with $\mathbf{E} \times \dot{\mathbf{B}}$ will not contribute to the current density. In the case where $\dot{\mathbf{B}}=0$, we have $F=B$ and Eq. (10) reduces to,

$\eta_{j}=\left(-\frac{1}{\tau_{R}}+a_{j} \Omega_{k} i\right) t, \quad I_{j}=\frac{-e^{-\left(-\frac{1}{\tau_{R}}+a_{j} \Omega_{k} i\right) t}}{B\left(-\frac{1}{\tau_{R}}+a_{j} \Omega_{k} i\right)}$.

Substituting Eq. (18) in master equations Eqs. (11)-(15), we obtain nonzero contribution for the current density from the terms with $\alpha_{i},(i=1,2,3,4)$. The coefficient $\alpha_{1}$ and $\alpha_{3}$ that relate to the electrical and Hall conductivities are defined in Eq. (16). The additional parameters that arise due to the time dependence of the external electric field take the following forms, 
$\alpha_{2}=\frac{q_{f_{k}} \epsilon\left[\left(\frac{\epsilon}{\tau_{R}}\right)^{2}-\left(q_{f_{k}} B\right)^{2}\right]}{\left[\left(\frac{\epsilon}{\tau_{R}}\right)^{2}+\left(q_{f_{k}} B\right)^{2}\right]^{2}}, \quad \alpha_{4}=\frac{2 q_{f_{k}}^{2} \epsilon^{2}}{\tau_{R}\left[\left(\frac{\epsilon}{\tau_{R}}\right)^{2}+\left(q_{f_{k}} B\right)^{2}\right]^{2}}$.

By employing Eq. (3) in Eq. (1), we can obtain an additional component to the Ohmic current due to the time dependence of the external electric field and can be quantified in terms of $\alpha_{2}$. Note that the additional component is higher-order in $\tau_{R}$ in comparison to the Ohmic current density. Similarly, $\alpha_{4}$ is related to the additional component to the Hall current in the presence of timevarying electric field. The results obtained are consistent with that of Ref. [33].

\section{Case III: Response to time-varying electromagnetic field}

In the case where both electric and magnetic fields are dependent on time, the contributions to the current density from all the terms associated with $\alpha_{i},(i=1,2,3,4,5)$ need to be considered systematically. We begin with evaluating the integrals described in Eq. (10) by choosing a particular time dependence of the external magnetic field. We consider the magnetic field with the form $\mathbf{B}=B_{0} e^{-\frac{t}{\tau_{B}}} \hat{\mathbf{z}}$, where $B_{0}$ is its amplitude and $\tau_{B}$ is the decay time parameter $[24,36]$ such that $F=B \sqrt{1+\frac{\tau_{R}}{\tau_{B}}}$, in the current analysis. Within the limit that the cyclotron frequency $\Omega_{k}$ is approximately equal to the decay frequency $\left(\tau_{B}^{-1}\right)$ of the magnetic field, Eq. (10) reduces to the following form,

$$
\begin{gathered}
\eta_{j}=-\frac{t}{\tau_{R}}+a_{j}\left(i \frac{\left.\sqrt{1+\frac{\tau_{R}}{\tau_{B}}} t\right),}{\tau_{B}} t\right) \\
I_{j}=\frac{1}{\sqrt{1+\frac{\tau_{R}}{\tau_{B}}} B_{0}} \frac{e^{\left(\frac{1}{\tau_{R}}+\frac{1}{\tau_{B}}-a_{j} i \frac{\sqrt{1+\frac{\tau_{R}}{\tau_{B}}}}{\tau_{B}}\right) t}}{\left(\frac{1}{\tau_{R}}+\frac{1}{\tau_{B}}-a_{j} i \frac{\sqrt{1+\frac{\tau_{R}}{\tau_{B}}}}{\tau_{B}}\right)} .
\end{gathered}
$$

Further, we proceed with the estimation of all $\alpha_{i}$ coefficients by substituting Eq. (20) and Eq. (21) in Eq. (11)-(15). Incorporating the nonzero contributions associated with $\alpha_{i},(i=1,2, . .5)$ in Eq. (4), we obtain five components of the induced current $\mathbf{j}=j_{e} \hat{\mathbf{e}}+j_{H}(\hat{\mathbf{e}} \times \hat{\mathbf{b}})$ as follows,

$$
j_{e}=j_{e}^{(0)}+j_{e}^{(1)}, \quad j_{H}=j_{H}^{(0)}+j_{H}^{(1)}+j_{H}^{(2)},
$$

where $j_{e}$ corresponds to the electric current in the direction of the electric field $\hat{\mathbf{e}}$ and $j_{H}$ is the electrical current in the direction perpendicular to both electric and magnetic fields $(\hat{\mathbf{e}} \times \hat{\mathbf{b}})$ with

$$
\begin{array}{r}
j_{e}^{(0)}=\frac{2 E}{3} N_{c} \sum_{k} \sum_{f}\left(q_{f k}\right)^{2} \int d P \frac{p^{2}}{\epsilon^{2}}\left(-\frac{\partial f_{k}^{0}}{\partial \epsilon}\right) M_{1}, \\
j_{e}^{(1)}=\frac{2 \dot{E}}{3} N_{c} \sum_{k} \sum_{f}\left(q_{f k}\right)^{2} \int d P \frac{p^{2}}{\epsilon^{2}} \frac{\partial f_{k}^{0}}{\partial \epsilon} M_{2}, \\
j_{H}^{(0)}=\frac{2 E}{3} N_{c} \sum_{k} \sum_{f}\left(q_{f k}\right)^{3} \int d P \frac{p^{2}}{\epsilon^{3}}\left(-\frac{\partial f_{k}^{0}}{\partial \epsilon}\right) M, \\
j_{H}^{(1)}=\frac{2 \dot{E}}{3} N_{c} \sum_{k} \sum_{f}\left(q_{f k}\right)^{3} \int d P \frac{p^{2}}{\epsilon^{3}} \frac{\partial f_{k}^{0}}{\partial \epsilon} M_{3},
\end{array}
$$

$j_{H}^{(2)}=\frac{2 E}{3 \tau_{B}} N_{c} \sum_{k} \sum_{f}\left(q_{f k}\right)^{3} \int d P \frac{p^{2}}{\epsilon^{3}}\left(-\frac{\partial f_{k}^{0}}{\partial \epsilon}\right) \tau_{R} M$,

where $\dot{E}=|\dot{\mathbf{E}}|$ and $M_{j}(j=1,2,3)$ functions can be defined as $M_{1}=\left(\frac{1}{\tau_{R}}+\frac{1}{\tau_{B}}\right) M, \quad M_{2}=-\left(\tau_{R} M_{1}-\frac{\tau_{R}^{2}}{\tau_{B}^{2}} M\right) /\left(1+\left(\frac{\tau_{R}}{\tau_{B}}\right)^{2}\right)$ and $M_{3}=\left(\tau_{R} M+\tau_{R}^{2} M_{1}\right) /\left(1+\left(\frac{\tau_{R}}{\tau_{B}}\right)^{2}\right)$ with,

$$
M=\left[\frac{1}{\tau_{R}}+\frac{1}{\tau_{B}}+\frac{\sqrt{1+\frac{\tau_{R}}{\tau_{B}}}}{\tau_{B}}\right]^{-1} .
$$

In Eqs. (23)-(27), $j_{e}^{(0)}$ denotes the leading-order Ohmic current and $j_{e}^{(1)}$ is the correction to the Ohmic current due to time dependence of the fields. The current $j_{H}^{(0)}$ is the Hall current in the medium generated due to the perpendicular electric and magnetic fields, and $j_{H}^{(1)}$ and $j_{H}^{(2)}$ are the correction to Hall current that comes from the terms $(\dot{\mathbf{E}} \times \mathbf{B})$ and $(\mathbf{E} \times \dot{\mathbf{B}})$, respectively.

\section{EFFECTS OF QCD MEDIUM INTERACTIONS AND ANISOTROPY}

The hot QCD equation of state (EoS) effect can be incorporated in the analysis through the quasiparticle description of the QCD medium [37]. In the present analysis, we employ the EQPM in which the thermal medium interactions are captured by a temperature-dependent fugacity parameter [34]. Considering the fact that large anisotropies arise due to the rapid expansion of the QGP, especially in the initial stages of heavy-ion collisions, the response of the anisotropic medium to the electromagnetic fields needs to be studied. The momentum anisotropy has been seen to have a visible impact on the response of the medium to the constant external fields [38]. We explore the impacts of thermal interaction and momentum anisotropy of the medium to the electric charge transport in the presence of time-varying fields below. 


\section{A. EoS effect: Quasiparticle description}

Within the EQPM prescription, the thermal QCD medium can be described in terms of noninteracting/ weakly interacting quasiparticles with the EQPM effective degrees of freedom and modified single-particle energy dispersion as [34],

$$
f_{k}^{0}=\frac{z_{k} e^{\frac{-\epsilon \mp \mu}{T}}}{1 \mp z_{k} e^{\frac{-\epsilon \mp \mu}{T}}}, \quad \omega_{k}=\epsilon+\delta \omega_{k},
$$

where $z_{k}$ is the effective fugacity parameter and is related to medium modified part of the energy dispersion as $\delta \omega_{k}=T^{2} \partial_{T} \ln z_{k}$. The near-equilibrium dynamics of the thermal medium can be described within the effective kinetic theory based on the EQPM [39]. The mean-field force term in the effective transport equation, which emerges from in-medium interactions, indeed appears as the mean-field contribution to the transport coefficients associated with the dissipative process. Following the prescriptions of the EQPM kinetic theory, we have the following forms for the components of current density:

$$
\begin{array}{r}
j_{e}^{(0)}=E \sum_{k} \sum_{f}\left(q_{f_{k}}\right)^{2} \int d \tilde{P}_{1 k}\left\{M_{1}-\delta \omega_{k} \frac{M_{1}}{p}\right\}, \\
j_{e}^{(1)}=-\dot{E} \sum_{k} \sum_{f}\left(q_{f_{k}}\right)^{2} \int d \tilde{P}_{1 k}\left\{M_{2}-\delta \omega_{k} \frac{M_{2}}{p}\right\}, \\
j_{H}^{(0)}=E \sum_{k} \sum_{f}\left(q_{f_{k}}\right)^{3} \int d \tilde{P}_{1 k}\left\{\frac{M}{\epsilon}-\delta \omega_{k} \frac{M}{p \epsilon}\right\}, \\
j_{H}^{(1)}=-\dot{E} \sum_{k} \sum_{f}\left(q_{f_{k}}\right)^{3} \int d \tilde{P}_{1 k}\left\{\frac{M_{3}}{\epsilon}-\delta \omega_{k} \frac{M_{3}}{p \epsilon}\right\}, \\
j_{H}^{(2)}=\frac{E}{\tau_{B}} \sum_{k} \sum_{f}\left(q_{f_{k}}\right)^{3} \int d \tilde{P}_{1 k} \tau_{R}\left\{\frac{M}{\epsilon}-\delta \omega_{k} \frac{M}{p \epsilon}\right\},
\end{array}
$$

where $d \tilde{P}_{1 k}=d P \frac{2 N_{c}}{3 \omega_{k}} \frac{p^{2}}{\epsilon}\left(-\frac{\partial f_{k}^{0}}{\partial \epsilon}\right)$. The term associated with $\delta \omega_{k}$ represents the mean-field correction term to each component of the current density. Note that at asymptotically high temperature, the medium behaves as an ultrarelativistic system with ideal EoS with $z_{k} \rightarrow 0$ and hence, the mean field contribution vanishes.

\section{B. Effect of momentum anisotropy}

The physics of anisotropy can be embedded in momentum distribution functions and can be represented in terms of rescaled isotropic distribution as [40,41],

$$
f_{(\text {aniso })_{k}}=\sqrt{1+\xi} f_{k}^{0}\left(\sqrt{p^{2}+\xi(\mathbf{p} \cdot \mathbf{n})^{2}}\right),
$$

where $\xi$ is the anisotropic parameter and $\mathbf{n}$ is the direction of anisotropy. For a weakly anisotropic medium, we have $\xi \ll 1$, and Eq. (35) reduces to,

$$
f_{(\text {aniso })_{k}}=f_{k}^{0}-\frac{\xi}{2 \epsilon T}(\mathbf{p} \cdot \mathbf{n})^{2} f_{k}^{02} e^{\frac{\epsilon \mp \mu}{T}},
$$

with $\mathbf{p}=(p \sin \theta \cos \phi, p \sin \theta \sin \phi, p \cos \theta)$ and we consider $\mathbf{n}=(\sin \alpha, 0, \cos \alpha)$ for the current analysis. The angles $\theta$ and $\phi$ denote spherical angles, and $\alpha$ denotes the projection of the anisotropic vector (define the angle between the anisotropic vector and $\mathbf{z}$-axis). The above choice of $\mathbf{n}$ indicates that the anisotropy is in the $\mathbf{x z}$ plane. Note that one can consider anisotropy in yz plane by choosing $\mathbf{n}=(0, \sin \alpha, \cos \alpha)$. Following the formalism of Ref. [38], and solving the relativistic Boltzmann equation we obtain the component of current density in the direction of an external time-varying electric field $(\hat{\mathbf{e}})$ in an anisotropic medium as,

$$
\left(j_{e}\right)_{\text {aniso }}=j_{e}^{(0)}+\delta j_{e}^{(0)}+j_{e}^{(1)}+\delta j_{e}^{(1)},
$$

where $j_{e}^{(0)}$ and $j_{e}^{(1)}$ are the isotropic components defined in Eq. (23) and Eq. (24), respectively. The corrections to the electric current due to momentum anisotropic of the medium, denoted by $\delta j_{e}^{(0)}$ and $\delta j_{e}^{(1)}$, can be defined as,

$$
\begin{gathered}
\delta j_{e}^{(0)}=-\xi \frac{E}{3} N_{c} \sum_{k} \sum_{f}\left(q_{f_{k}}\right)^{2} l \int_{0}^{\infty} d p L e^{\frac{\epsilon \mp \mu}{T}} M_{1}, \\
\delta j_{e}^{(1)}=\xi \frac{\dot{E}}{3} N_{c} \sum_{k} \sum_{f}\left(q_{f_{k}}\right)^{2} l \int_{0}^{\infty} d p L e^{\frac{\epsilon \mp \mu}{T}} M_{2},
\end{gathered}
$$

with $l=\frac{1}{6 \pi^{2} T^{2}}$ and $L=\frac{p^{6}}{\epsilon}\left(f_{k}^{0}\right)^{2}$. It is important to emphasize that in the absence of magnetic field Eq. (38) reduces back to the findings of [38]. Similarly, we define the components of current density in the direction transverse to the fields $[(\hat{\mathbf{e}} \times \hat{\mathbf{b}})]$ in the anisotropic medium as,

$$
\left(j_{H}\right)_{\text {aniso }}=j_{H}^{(0)}+\delta j_{H}^{(0)}+j_{H}^{(1)}+\delta j_{H}^{(1)}+j_{H}^{(2)}+\delta j_{H}^{(2)} .
$$

The isotropic terms are described in Eqs. (25)-(27) and the anisotropic contributions take the following forms,

$$
\begin{gathered}
\delta j_{H}^{(0)}=-\xi \frac{E}{3} N_{c} \sum_{k} \sum_{f}\left(q_{f_{k}}\right)^{3} l \int_{0}^{\infty} d p \frac{L}{\epsilon} e^{\frac{\epsilon \mp \mu}{T}} M, \\
\delta j_{H}^{(1)}=\xi \frac{\dot{E}}{3} N_{c} \sum_{k} \sum_{f}\left(q_{f_{k}}\right)^{3} l \int_{0}^{\infty} d p \frac{L}{\epsilon} e^{\frac{\epsilon \mp \mu}{T}} M_{3}, \\
\delta j_{H}^{(2)}=-\xi \frac{E}{3 \tau_{B}} N_{c} \sum_{k} \sum_{f}\left(q_{f_{k}}\right)^{3} l \int_{0}^{\infty} d p \frac{L \tau_{R}}{\epsilon} e^{\frac{\epsilon \mp \mu}{T}} M .
\end{gathered}
$$




\section{RESULTS AND DISCUSSIONS}

We initiate the discussion with the QCD medium response to the time-varying electromagnetic field. The medium response to the fields is quantified in terms of induced current in the direction of the external electric field $j_{e}$ and in the direction perpendicular to electromagnetic fields $j_{H}$. We define the following ratios,

$$
R_{e}=\frac{j_{e}^{(0)}}{E T}+\frac{j_{e}^{(1)}}{E T}, \quad R_{H}=\frac{j_{H}^{(0)}}{E B T}+\frac{j_{H}^{(1)}}{E B T}+\frac{j_{H}^{(2)}}{E B T},
$$

such that $j_{e}^{(0)} /(E T)=\sigma_{e} / T$ denotes the dimensional less quantity in the case of constant electromagnetic fields where the term $j_{e}^{(1)} /(E T)$ gives further correction due to the time dependence of E. Similarly, $j_{H}^{(0)} /(E B T)$ represents the leading order term $\sigma_{H} /(B T)$ followed by the correction terms with $j_{H}^{(1)}$ and $j_{H}^{(2)}$ in the direction $(\hat{\mathbf{e}} \times \hat{\mathbf{b}})$. The spacetime profile of the electric and magnetic fields are described in Ref. [24] in which the strength of the inhomogeneity in time can be quantified with decay time $\tau_{E / B}$ as $\dot{\mathbf{E}} / \mathbf{E} \propto \tau_{E}$ and $\dot{\mathbf{B}} / \mathbf{B} \propto \tau_{B}$.

The impact of the time dependence of the external fields on the temperature behavior of $R_{e}$ is depicted in Fig. 1 (left panel). We observe that the time dependence of the electric and magnetic fields has a significant impact on the medium response of the system. In the case of a constant magnetic field and time-varying electric field, $R_{e}$ is higher than that in the case of constant electric and magnetic field. The increment is due to the extra component of electrical current density associated with $\dot{\mathbf{E}}$ as described in Eq. (4). Unlike in the case of a time-varying electric field, the inclusion of time dependence of the magnetic field decreases the value $R_{e}$ for the chosen temperature range.
Note that the $\alpha_{i}=0$ for $i=(6,7,8)$ due to the parity symmetry in the analysis, which indicates that the contribution from the components associated with $\mathbf{B}, \dot{\mathbf{B}}$ vanishes in the present analysis. It is important to emphasize that these components may have a significant role in a chiral medium. However, the time dependence of magnetic field enters in the analysis of electric current density through the $\alpha_{1}$ and $\alpha_{2}$ (i.e, through the components associated with $\mathbf{E}$ and $\dot{\mathbf{E}}$ ). The general forms of $\alpha_{1}$ and $\alpha_{2}$ in a time-varying electromagnetic field are described in Eq. (11) and Eq. (14), respectively. We compare the results with the lattice estimation of $\sigma_{e} / T$ at vanishing magnetic field and constant electric field as described in Refs. $[32,42,43]$ and also with the transport theory results [15]. Notably, the effect of inhomogeneity of time of the fields to the current density is seen to be more pronounced in the temperature regime closer to the transition temperature $T_{c}$. The effect of additional components to the Hall current due to the time dependence of the fields is studied by demonstrating the temperature dependence of $R_{H}$ in Fig. 1 (right panel). Similar to the case of $j_{e} \hat{\mathbf{e}}$, the inclusion of time dependence of the electric and magnetic fields has a visible impact on the Hall current, especially in the lowtemperature regimes. It is also important to note that unlike in the case of electrical current density, the Hall current has a nonzero contribution from the component associated with the time dependence of magnetic field $(\mathbf{E} \times \dot{\mathbf{B}})$ in addition to the dependence of the time varying magnetic field on $\alpha_{3}$ and $\alpha_{4}$.

The impact of the momentum anisotropy on the temperature behavior of $R_{e}$ and $R_{H}$ are plotted in Fig. 2. We observe both $j_{e} \hat{\mathbf{e}}$ and $j_{H}(\hat{\mathbf{e}} \times \hat{\mathbf{b}})$ in the presence of timevarying fields decrease with an increase in anisotropy in the medium. This observation agrees with the study of charge transport with constant electromagnetic fields [38].

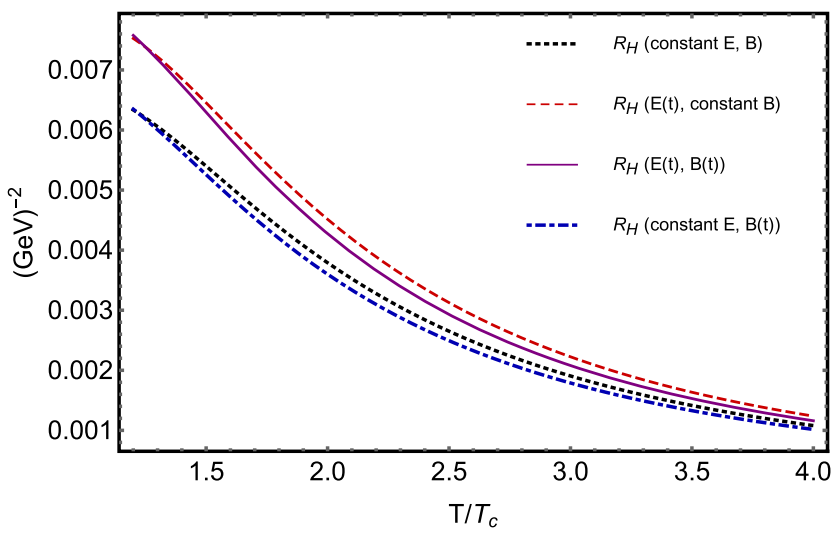

FIG. 1. The temperature dependence of $R_{e}$ (left panel) and $R_{H}$ (right panel) for various choices of the external electromagnetic fields: (i). (Constant E, B), (ii). Time-varying electric and constant magnetic field (E(t), constant B), (iii). Constant electric and time-varying magnetic field [constant $\mathrm{E}, \mathrm{B}(\mathrm{t})$ ], (iv). Time-varying electromagnetic fields $[\mathrm{E}(\mathrm{t}), \mathrm{B}(\mathrm{t})]$. For constant magnetic field case $e B=$ $0.03 \mathrm{GeV}^{2}$ and we choose $\tau_{B}=9 \mathrm{fm}, \tau_{E}=7 \mathrm{fm}$ for the time-varying cases. The results are compared with lattice data [32,42,43] and transport theory estimation [15] at $B=0$. 

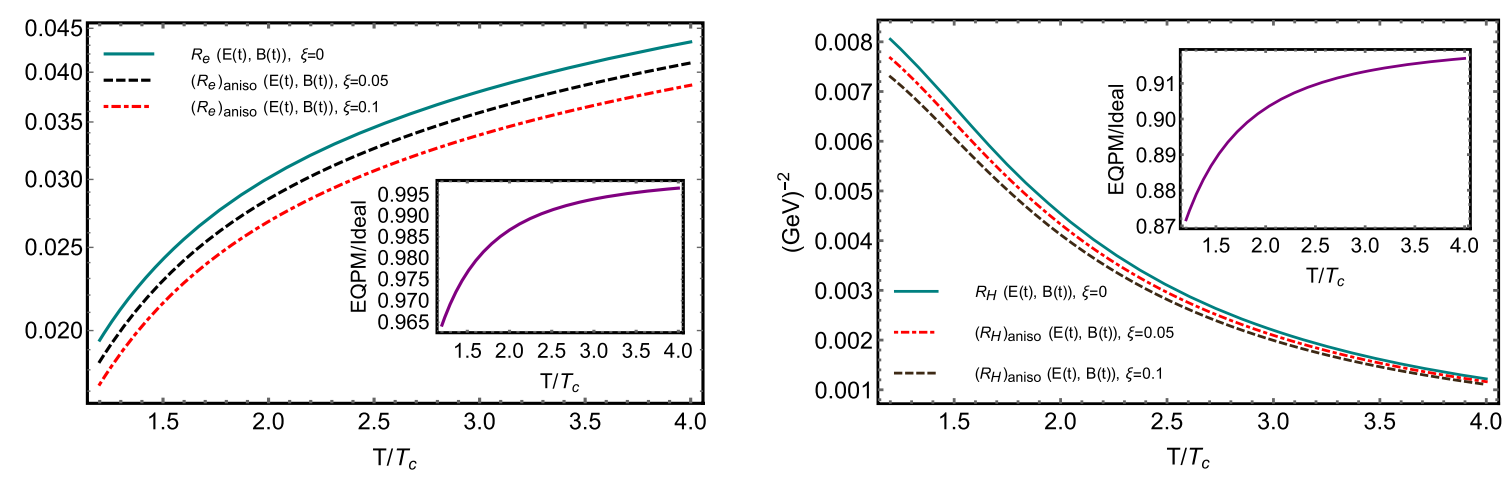

FIG. 2. Effect of anisotropy and QCD EoS on the temperature behavior of $R_{e}$ (left panel) and $R_{H}$ (right panel) in the case of timevarying fields with $\tau_{B}=9 \mathrm{fm}$. $\left(R_{e}\right)_{\text {aniso }}$ and $\left(R_{H}\right)_{\text {aniso }}$ denote $R_{e}$ and $R_{H}$ in an anisotropic medium.

We have also explored the effect of in-medium interactions on the charge transport in the QCD medium (inset plots). It is seen that the QCD EoS and the mean-field effects further decrease the current densities. The effect of the EoS is prominent at the low-temperature regimes, especially for the Hall current density.

\section{CONCLUSION AND OUTLOOK}

In conclusion, we have explored the response of the QCD medium to the time-varying electromagnetic fields. We have obtained a general form of the near-equilibrium distribution function of the medium constituents in the presence of inhomogeneous electromagnetic fields. To that end, we have solved the relativistic Boltzmann equation within the relaxation time approximation. The QCD medium response to the external time dependent fields has been quantified in terms of induced current. Notably, we have obtained additional components to the electrical and Hall current densities that arise from the time dependence of the fields. The impact of the additional terms on the current densities and the respective conductivities are observed to be significant, especially in the temperature regime not very far from $T_{c}$. We have compared the results with the lattice estimations and transport theory results with constant external fields. Further, we have studied the impacts of the thermal QCD EoS and momentum anisotropy to the electric charge transport in the presence of time-varying fields. The in-medium interaction effects are incorporated in the analysis through the quasiparticle and the followed effective kinetic theory description of the charge transport. It is seen that both the EoS and anisotropic effects to the current densities are non-negligible in the temperature regime near $T_{c}$.

The additional components to the Ohmic and Hall current densities due to the decay of the electric and magnetic fields in the medium may perhaps play a significant role in the realistic magnetohydrodynamical framework for the QCD medium in the heavy-ion collision experiments. Furthermore, the inclusion of time dependence of the electromagnetic field along with the impact of momentum anisotropy of the medium to charge transport within an effective description is essential for the complete understanding of charge-dependent directed flow of final-stage particles in the asymmetric collision experiments [44]. We intend to explore the phenomenological aspect of the analysis in the near future. The study of thermal and momentum transport in the presence of a timevarying magnetic field in the QCD medium is another interesting direction to explore in the future.

\section{ACKNOWLEDGMENTS}

M. K. would like to acknowledge the Indian Institute of Technology Gandhinagar for the postdoctoral fellowship.

\section{APPENDIX: NEAR-EQUILIBRIUM DISTRIBUTION FUNCTION}

The nonequilibrium part of the distribution function in the presence of an external electromagnetic field can be obtained by solving the Boltzmann equation. The covariant form of the Boltzmann equation takes the following form,

$$
p^{\mu} \partial_{\mu} f_{k}(x, p)+q_{f_{k}} F^{\mu \nu} p_{\nu} \partial_{\mu}^{(p)} f_{k}=C\left(f_{k}\right),
$$

where $C\left(f_{k}\right)$ is the collision kernel that takes the following form in the relaxation time approximation as,

$$
C_{k}=-(u \cdot p) \frac{\delta f_{k}}{\tau_{R}},
$$

with $\tau_{R}$ is the thermal relaxation time. Here, we have employed the following four-vector notations: The quantity $u^{\mu}=(1,0)$ is the fluid velocity, $p^{\mu}=\left(p^{0}, \mathbf{p}\right)$ and $F^{\mu \nu}$ is the electromagnetic strength tensor. By employing the relations, $F^{0 i}=E^{i}$ and $2 F_{i j}=\epsilon_{i j k} B^{k}$ one can define the force term due to the external electromagnetic fields as follows,

$$
\mathcal{F}^{\mu}=q_{f_{k}} F^{\mu \nu} p_{\nu}=\left(p^{0} \mathbf{v} \cdot \mathcal{F}, p^{0} \mathcal{F}\right),
$$


where $\mathcal{F}=q_{f_{k}}(\mathbf{E}+\mathbf{v} \times \mathbf{B})$. To represent the Boltzmann equation in terms of three vector notation, we need to substitute the above-defined definitions of the four vectors in the Boltzmann equation described in Eq. (A1). Hence, we have

$$
\begin{aligned}
& p^{0} \partial_{0} f_{k}+p^{i} \partial_{i} f_{k}+\mathcal{F}^{0} \partial_{0}^{(p)} f_{k}+\mathcal{F}^{i} \partial_{i}^{(p)} f_{k}=-p^{0} \frac{\delta f_{k}}{\tau_{R}}, \\
& \frac{\partial f_{k}}{\partial t}+\mathbf{v} \cdot \frac{\partial f_{k}}{\partial \mathbf{x}}+\frac{\mathcal{F} \cdot \mathbf{v}}{p^{0}} \frac{\partial f_{k}}{\partial p^{0}}+\mathcal{F} \cdot \frac{\partial f_{k}}{\partial \mathbf{p}}=-\frac{\delta f_{k}}{\tau_{R}} .
\end{aligned}
$$

Employing the chain rule of differentiation $\frac{\partial p^{0}}{\partial \mathbf{p}} \frac{\partial}{\partial p^{0}}+\frac{\partial}{\partial \mathbf{p}} \rightarrow$ $\frac{\partial}{\partial \mathbf{p}}$ in Eq. (A4), the Boltzmann equation takes the form as follows,

$$
\frac{\partial f_{k}}{\partial t}+\mathbf{v} \cdot \frac{\partial f_{k}}{\partial \mathbf{x}}+q_{f_{k}}[\mathbf{E}+\mathbf{v} \times \mathbf{B}] \cdot \frac{\partial f_{k}}{\partial \mathbf{p}}=-\frac{\delta f_{k}}{\tau_{R}} .
$$

Next, we need to consider possible components of the current density that are linear in terms of the external electromagnetic field. Following Ref. [36], we have considered the possible components that are proportional to $\mathbf{E}, \dot{\mathbf{E}},(\mathbf{E} \times \mathbf{B}),(\dot{\mathbf{E}} \times \mathbf{B}),(\mathbf{E} \times \dot{\mathbf{B}}),(\boldsymbol{\nabla} \times \mathbf{E}), \mathbf{B}, \dot{\mathbf{B}},(\boldsymbol{\nabla} \times \mathbf{B})$. Note that we have considered the case in which the electromagnetic field varies slowly in space and time (i.e., for larger value of $\tau_{E}$ and $\tau_{B}$ ) and hence neglected the terms with two and higher space-time derivatives. As the parity property of the current density is different from those of $\mathbf{B}, \dot{\mathbf{B}},(\boldsymbol{\nabla} \times \mathbf{E})$, the components of current density associated with these quantities will exist only for a chiral medium where the chiral chemical potential $\mu_{5} \neq 0$. It is important to emphasize that the Maxwell's equations further simplify the expansion as terms $(\boldsymbol{\nabla} \times \mathbf{E})$ and $\dot{\mathbf{B}}$ are related by Maxwell's equations. To solve Eq. (A5), we consider an ansatz to express the deviation of the distribution function from the equilibrium in terms of possible sources of perturbation due to electromagnetic fields as (for the system with $\mu_{5}=0$ ),

$$
\delta f_{k}=(\mathbf{p} . \boldsymbol{\Xi}) \frac{\partial f_{k}^{0}}{\partial \epsilon}
$$

where

$$
\begin{aligned}
\boldsymbol{\Xi}= & \alpha_{1} \mathbf{E}+\alpha_{2} \dot{\mathbf{E}}+\alpha_{3}(\mathbf{E} \times \mathbf{B})+\alpha_{4}(\dot{\mathbf{E}} \times \mathbf{B})+\alpha_{5}(\mathbf{E} \times \dot{\mathbf{B}}) \\
& +\alpha_{6}(\boldsymbol{\nabla} \times \mathbf{E}),
\end{aligned}
$$

where the unknown coefficients $\alpha_{i}$ can be obtained by solving the Boltzmann equation. However, for a more general case one needs to consider the quadratic terms in electric field such as $\boldsymbol{\nabla}\left(\mathbf{E}^{2}\right), \mathbf{E}(\boldsymbol{\nabla} \cdot \mathbf{E}),(\mathbf{E} \cdot \boldsymbol{\nabla}) \mathbf{E}$, and also in magnetic field. For the current analysis, we have focused on the leading order contributions (linear in electromagnetic fields and their time derivatives). A more general analysis is beyond the scope of the present analysis. This method has been employed in the case of the constant electromagnetic field in Refs. [13,45], and is also widely used in the estimation of nonequilibrium part of distribution function for the study of thermoelectric effect [46-48] (where the temperature gradient is the source term instead of the electric field) in a magnetized QCD medium.
[1] J. Adams et al. (STAR Collaboration), Nucl. Phys. A757, 102 (2005).

[2] A. Jaiswal et al., Int. J. Mod. Phys. E 30, 2130001 (2021).

[3] S. Acharya et al. (ALICE Collaboration), Phys. Rev. Lett. 125, 022301 (2020).

[4] J. Adam et al. (STAR Collaboration), Phys. Rev. Lett. 123, 162301 (2019).

[5] K. Tuchin, Phys. Rev. C 88, 024911 (2013).

[6] L. McLerran and V. Skokov, Nucl. Phys. A929, 184 (2014).

[7] E. Stewart and K. Tuchin, Nucl. Phys. A1016, 122308 (2021).

[8] K. Tuchin, Phys. Rev. C 102, 014908 (2020).

[9] L. Yan and X.-G. Huang, arXiv:2104.00831.

[10] Y. Hirono, M. Hongo, and T. Hirano, Phys. Rev. C 90, 021903 (2014).

[11] K. Fukushima, D. E. Kharzeev, and H. J. Warringa, Phys. Rev. D 78, 074033 (2008).

[12] Y. Yin, Phys. Rev. C 90, 044903 (2014).
[13] B. Feng, Phys. Rev. D 96, 036009 (2017).

[14] A. Das, H. Mishra, and R. K. Mohapatra, Phys. Rev. D 99, 094031 (2019).

[15] L. Thakur and P. Srivastava, Phys. Rev. D 100, 076016 (2019).

[16] J. Dey, S. Satapathy, P. Murmu, and S. Ghosh, Pramana 95, 125 (2021).

[17] M. Kurian, Phys. Rev. D 102, 014041 (2020).

[18] A. Dash, S. Samanta, J. Dey, U. Gangopadhyaya, S. Ghosh, and V. Roy, Phys. Rev. D 102, 016016 (2020).

[19] K. Hattori, S. Li, D. Satow, and H.-U. Yee, Phys. Rev. D 95, 076008 (2017).

[20] K. Fukushima and Y. Hidaka, Phys. Rev. Lett. 120, 162301 (2018).

[21] M. Kurian and V. Chandra, Phys. Rev. D 96, 114026 (2017).

[22] S. Ghosh, A. Bandyopadhyay, R. L. Farias, J. Dey, and G. a. Krein, Phys. Rev. D 102, 114015 (2020). 
[23] W.-T. Deng and X.-G. Huang, Phys. Rev. C 85, 044907 (2012).

[24] M. Hongo, Y. Hirono, and T. Hirano, Phys. Lett. B 775, 266 (2017).

[25] H. Li, X.-1. Sheng, and Q. Wang, Phys. Rev. C 94, 044903 (2016).

[26] X.-G. Huang, Rep. Prog. Phys. 79, 076302 (2016).

[27] M. Greif, I. Bouras, C. Greiner, and Z. Xu, Phys. Rev. D 90 , 094014 (2014).

[28] W. Cassing, O. Linnyk, T. Steinert, and V. Ozvenchuk, Phys. Rev. Lett. 110, 182301 (2013).

[29] A. Puglisi, S. Plumari, and V. Greco, Phys. Rev. D 90, 114009 (2014).

[30] R. Kubo, J. Phys. Soc. Jpn. 12, 570 (1957).

[31] N. Astrakhantsev, V. V. Braguta, M. D’Elia, A. Y. Kotov, A. A. Nikolaev, and F. Sanfilippo, Phys. Rev. D 102, 054516 (2020).

[32] A. Amato, G. Aarts, C. Allton, P. Giudice, S. Hands, and J.-I. Skullerud, Phys. Rev. Lett. 111, 172001 (2013).

[33] K. K. Gowthama, M. Kurian, and V. Chandra, Phys. Rev. D 103, 074017 (2021).

[34] V. Chandra and V. Ravishankar, Phys. Rev. D 84, 074013 (2011).

[35] A. Hosoya and K. Kajantie, Nucl. Phys. B250, 666 (1985).
[36] D. Satow, Phys. Rev. D 90, 034018 (2014).

[37] V. Mykhaylova and C. Sasaki, Phys. Rev. D 103, 014007 (2021).

[38] P. K. Srivastava, L. Thakur, and B. K. Patra, Phys. Rev. C 91, 044903 (2015).

[39] S. Mitra and V. Chandra, Phys. Rev. D 97, 034032 (2018).

[40] B. Schenke, M. Strickland, C. Greiner, and M. H. Thoma, Phys. Rev. D 73, 125004 (2006).

[41] P. Romatschke and M. Strickland, Phys. Rev. D 68, 036004 (2003).

[42] G. Aarts, C. Allton, J. Foley, S. Hands, and S. Kim, Phys. Rev. Lett. 99, 022002 (2007).

[43] H.-T. Ding, A. Francis, O. Kaczmarek, F. Karsch, E. Laermann, and W. Soeldner, Phys. Rev. D 83, 034504 (2011).

[44] U. Gürsoy, D. Kharzeev, E. Marcus, K. Rajagopal, and C. Shen, Phys. Rev. C 98, 055201 (2018).

[45] A. Das, H. Mishra, and R. K. Mohapatra, Phys. Rev. D 100, 114004 (2019).

[46] A. Das, H. Mishra, and R. K. Mohapatra, Phys. Rev. D 102, 014030 (2020).

[47] M. Kurian, Phys. Rev. D 103, 054024 (2021).

[48] H.-X. Zhang, J.-W. Kang, and B.-W. Zhang, Eur. Phys. J. C 81, 623 (2021). 